Article

\title{
Definition of Internet of Things (IoT) Cyber Risk - Discussion on a Transformation Roadmap for Standardization of Regulations, Risk Maturity, Strategy Design and Impact Assessment
}

\author{
Petar Radanliev 1, David Charles De Roure 1, Jason R.C. Nurse 2, Pete Burnap ${ }^{3}$, Eirini Anthi ${ }^{3}$, \\ Uchenna Ani ${ }^{4}$, La'Treall Maddox ${ }^{5}$, Omar Santos ${ }^{5}$ and Rafael Mantilla Montalvo ${ }^{5}$ \\ 1 Oxford e-Research Centre, Department of Engineering Sciences, University of Oxford, UK, \\ petar.radanliev@oerc.ox.ac.uk; david.deroure@oerc.ox.ac.uk; \\ 2 School of Computing, University of Kent, UK, j.r.c.nurse@kent.ac.uk; \\ 3 School of Computer Science and Informatics, Cardiff University, p.burnap@cs.cardiff.ac.uk; \\ 4 STEaPP, Faculty of Engineering Science, University College London, u.ani@ucl.ac.uk; \\ 5 Cisco Research Centre, Research Triangle Park, USA, lamaddox@cisco.com; osantos@cisco.com; \\ montalvo@cisco.com \\ * Corresponding author: Petar Radanliev: petar.radanliev@oerc.ox.ac.uk
}

\begin{abstract}
The Internet-of-Things (IoT) enables enterprises to obtain profits from data but triggers data protection questions and new types of cyber risk. Cyber risk regulations for the IoT however do not exist. The IoT risk is not included in the cyber security assessment standards, hence, often not visible to cyber security experts. This is concerning, because companies integrating IoT devices and services need to perform a self-assessment of its IoT cyber security posture. The outcome of such self-assessment needs to define a current and target state, prior to creating a transformation roadmap outlining tasks to achieve the stated target state. In this article, a comparative empirical analysis is performed of multiple cyber risk assessment approaches, to define a high-level potential target state for company integrating IoT devices and/or services. Defining a high-level potential target state represent is followed by a high-level transformation roadmap, describing how company can achieve their target state, based on their current state. The transformation roadmap is used to adapt IoT risk impact assessment with a Goal-Oriented Approach and the Internet of Things Micro Mart model.
\end{abstract}

Keywords: Internet of Things; Micro Mart model; Goal-Oriented Approach; transformation roadmap; Cyber risk regulations; empirical analysis; cyber risk self-assessment; cyber risk target state

\section{Introduction}

Economic impact of Internet-of-Things (IoT) cyber risk is increasing with the integration of digital infrastructure in the digital economy [1]. Cyber security standardisation and regulation would play a key role in the process of reducing cyber-attacks while continuing to harness the economic values.

The cyber risk from IoT devices is present across different and sometimes at a higher level in sectors where such risk is unexpected. For example, in the US, healthcare is now the largest target of cyber security attacks, reportedly at greater risk than manufacturing and banking [2]. According to the same report [2], the value of stolen personal health information is ten to twenty times greater than the value of a stolen credit card number. To understand and define a generic target state for cyber 
maturity, we need to understand the business context and the cyber risk priorities through discussions between cyber security experts and decision makers [3].

This research article conducts epistemological analysis focused on understanding the best approach for increasing safety, security and economic value in the IoT space. Our research has two objectives. To identify and capture a high-level target state for the mitigation of cyber risk from the IoT, and to adapt existing cyber security practices and standards to include IoT cyber risk in a highlevel cyber security transformation roadmap. We discuss and expand on these further in the remainder of this article. In Section 2 we present the research methodology.

\section{Methodology}

There is a strong interest in regulating the cyber risk assessment procedures. Regulation and standardisation of cyber security frameworks, models and methodologies has not been done until present. Standardisation in this article refers to the compounding of knowledge to advance the efforts on integrating cyber risk standards and governance, and to offer a better understanding of cyber risk assessments. Here we combine literature analysis with an empirical with a comparative analysis. The empirical analysis is conducted with seven cyber risk frameworks and two cyber risk models. The comparative analysis engages with fifteen high-tech national strategies.

\section{Current state of cyber risk}

Current cyber risk trends are based on risk from intelligent manufacturing equipment, artificial intelligence, the cloud, and IoT, creating risk from systems of machines capable of interacting with the cyber-physical world [4], [5]. The integration of new technologies creates cyber security risk, e.g. integrating less secured systems [6] in manufacturing [7] and supply chains [8]-[12]. Existing cyber risk assessment models [13]-[19] ignore the risk impacts of sharing infrastructure and the cyber risk estimated loss range variously [1]. Further cyber risk assessment challenges emerge from compiling of connected systems devices and platforms [4], [20]. The machines are becoming social CPS [21]-[23] operating as real-time IoT systems of systems [24], [25], creating cyber risk from data in transit. This requires standardisation of design and process [15], [26] because such system security is complex.

\section{Uncovering the best method to define a unified cyber risk assessment through a comparative empirical research study}

\subsection{Comparative study of IoT in Industry 4.0}

From this comparative study, the main IoT risk elements of each IoT strategy are compounded into categories representing the most prominent IoT cyber risk vectors (see Table 1). However, the compelling of data into these categories is quite challenging, as some strategies, represent a collection of descriptive explanations and do not provide explicit IoT cyber risk vectors. Such descriptive explanations present complexities in developing a unifying strategy. To resolve this issue, we use the grounded theory methodology, where most prominent IoT cyber risk vectors are categorised and used as reference themes. Then, the categories are used for examining IoT cyber risk, to define a standardisation approach that relates various IoT risk vectors to eliminate conflicts in different and sometimes contrasting assessments of risk vectors.

\subsection{Empirical analysis of gaps in cyber risk impact assessment approaches}

The empirical analysis aims to identify cyber security frameworks and to compare interlinkages with the IoT risk in the Industry 4.0. Some of the frameworks reviewed propose diverse qualitative methods, and some qualitative approaches for measuring cyber risk propose methodologies[27]-[31].

The most advanced cyber risk impact assessment framework is the US NIST [32]. The NIST framework argues that to reach the required cyber security maturity level, the current cyber state can be transformed into a given a target cyber state by applying a specific cyber security framework implementation guidance [33]. However, the NIST guidance is specifically designed for Federal 
Agencies and requires adaptations to be applicable to enterprises. The NIST framework requires determining a detailed current cyber profile and a maturity level but does not provide a model for these steps.

The CVSS calculator [34], [35] could be used to create a current cyber profile, in addition to the Exostar system [36] for determining the supply chain cyber maturity level, and CMMI [37] the overall current state of cyber maturity. The final gap in the NIST framework is the lack of cyber risk quantitative assessment, which is crucial for making an informed and detailed recommendations for a target cyber profile. The Factor Analysis of Information Risk Institute (FAIR) [38] aims to address this gap in the NIST framework. FAIR Institute adapts existing quantitative models, e.g. RiskLens [39], and Cyber VaR (CyVaR) [40]. In a way, FAIR is complementing the work of NIST and the International Organisation for Standardisation (ISO) [41], which is the international standard-setting body and includes cyber risk standards. Notable for this discussion, only FAIR [38] provides recommendations for quantitative risk estimation. To complete the risk assessment, the cyber risk from supply chains needs to be simplified [31], [42], [43]. To identify a current cyber risk state that includes supply chain cyber risks, the Exostar system [36], can be used for complimenting the CVSS and covering the supply chain aspect of cyber risk. Further analysis, including SWAT and GAP analysis are considered beyond the scope of this conference paper, but can be found in other articles [43]-[47].

\subsection{Transformation roadmap for standardisation of IoT risk impact assessment}

To define a transformation roadmap for standardisation of IoT risk impact assessment, the methodology follows recently established approaches [1], [4], [5], [44], [47]-[49]. The design principles define how to identify, manage, estimate, and prioritise cyber risk (Table 1).

\begin{tabular}{|l|l|l|l|l|l|l|l|}
\hline Vectors & FAIR & CMMI & CVSS & ISO & NIST & Octave & TARA \\
\hline $\begin{array}{l}\text { Risk } \\
\text { identification } \\
\text { (measure) }\end{array}$ & Financial & $\begin{array}{l}\text { Maturity } \\
\text { models }\end{array}$ & Base metrics & ISO 27032 & Categorising & Workshops & Threat Matrix \\
\hline $\begin{array}{l}\text { Risk } \\
\text { management } \\
\text { (standardise) }\end{array}$ & Compliance & $\begin{array}{l}\text { ISO 15504 - } \\
\text { SPICE }\end{array}$ & $\begin{array}{l}\text { Mathematical } \\
\text { approximation }\end{array}$ & ISO 27001 & Assembling & Repeatability Template \\
threats
\end{tabular}

Table 1: High level target state for standardisation of IoT risk impact assessment

In Table 1, the resulting definition of transformation roadmap are reduced in content with the controlled convergence methods [31].

\section{Case study research}

\subsection{The case study}

The case study research involved four workshops that included 18 distinguished engineers from Cisco Systems, and 2 distinguished engineers from Fujitsu. The workshops with Cisco Systems were conducted in the USA in four different Cisco research centres. These centres were as follows: First Centre - Security and Trust Organisation; Second Centre - Advanced Services; Third Centre - Security Business Group; and Fourth Centre - Cisco Research Centre. In pursuit of validity, a separate workshop was conducted with two experts from Fujitsu centre for Artificial Intelligence the UK. The Fujitsu workshop was conducted separately to avoid those experts being influenced or outspoken by the larger group from Cisco systems.

The first two Cisco workshops were conducted to apply the controlled convergence [9], [31]. This approach to pursuing validity follows existing literature on this topics [50], [51] and provides 
clear definitions that specify the units of analysis for IoT cyber risk vectors. The IoT risk units of analysis from individual IoT strategy are combined into standardisation vectors. The process of defining the standardisation vectors followed the controlled convergence method [52], [53], where experts were asked to confirm the valid concept, merge duplicated concepts, and delete conflicting concepts. The main limitation of the Pugh controlled convergence method is the difficulty in gathering large number of experts in one location.

\subsection{Transformation imperatives describing how IoT companies can achieve their target state based on their current state with a Goal-Oriented approach}

Following the identification of a high-level target state in the comparative empirical analysis, the controlled convergence was applied to narrow the emerging implementation tasks through case study. The controlled convergence was applied for the development of a transformation roadmap for the high-level target state (Table 1). To build the transformation roadmap, the emerging categories are validated with applying the controlled convergence with a group of experts. The process of confirming validity of the data in Table 2, outlines the transformation process but does not include all the steps as the aim is to present a methodology, not the actions. The rationale is that different enterprises will have different cyber security steps to perform in order to transition to a higher maturity lever. A long and detailed list of steps can be found in some of our work in progress articles [42], [43], [46].

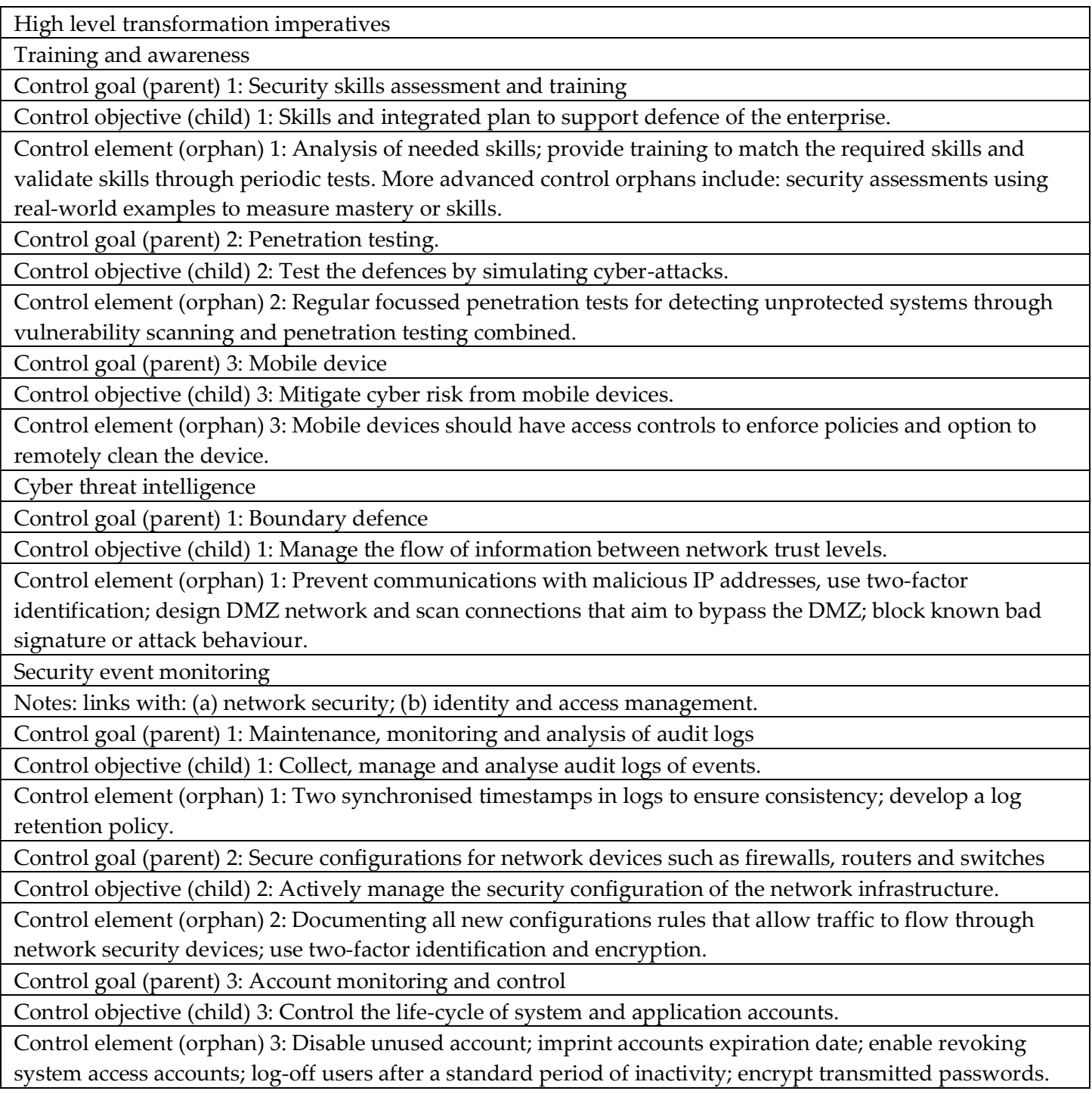


Table 2: Transformation roadmap- describing examples of how IoT companies can achieve their target state based on their current state with a Goal-Oriented approach

For implementing the recommendations emerging from the transformational imperatives, we refer to the NIST cyber security implementation tiers as support guidance. These implementation steps are the prime focus of government and industry efforts for improving cyber security exposure. In the following section, the transformation roadmap and imperatives can be used to define a new IoT risk impact assessment with a goal-oriented approach and the Internet of Things Micro Mart model.

\section{IoT risk impact assessment with the transformation roadmap and imperatives}

Dependency goal-oriented modelling can be applied to connect unconnected risk models and to build a risk model for a complex IoT systems. The first step is to link separate models. This requires identifying the shared principles from the multiple models that we are connecting. Then, to determine the level of dependency risk, we need to understand the dependencies of the shared principles.

\subsection{IoT Risk Analysis through Functional Dependency}

Dependency modelling and analysis provides a means to support the management of functional and operational complexities within IoT systems with focus on the system elements, measures of a design or operational challenge, as well as the functional dependencies that define their associations. Dependency modelling and analysis can support a superior understanding of connectivity and its implications on performance, and can assist in constructing, improving, and maintaining of such complex system. The construct and exchanges that happen in IoT domain defines a tightly coupled association amongst constituting components and sub-systems such considerably on the correct functions of another linked component or system. This is considered a dependency relationship, and can either be direct (a first order dependency) or indirect (a subsequent higher order dependency) [54]. For example, from a typical IoT architecture [55], normal functions for components and services on the application layer typically depend on the normal functioning of their counterparts on the network layer. This latter also relies on the perception layer component and services. If a component or service on the perception layer is compromised, such impairment can alter the correct functioning of connected component or service on the network or application layers. Thus, security risks in an IoT domain may not exactly be drawn from the failure of one specific IoT component, but most often extend to the failure of other IoT components that can be recipients of rippling impacts. This dependency amongst IoT sub-systems and components can also cause impacts or failures to cascade from one affected system or component onto another; worsening the damaging impacts [56], [57]. One way of achieving IoT dependency modelling is through a network-based functionality dimension - analysing how the functionality of one system or component can affect the functionalities of other systems or components [58], explorable on the basis of connectivity and process configurations over multi-layered IoT architecture. Graph theory [54], [59] - using vertices and edges; provides a way to simply represent (directly or logically) the dependency of IoT components within and across multiple functionality layers.

\subsection{IoT risk impact assessment with a Goal-Oriented Approach}

Identifying shared principles for multiple independent cyber risk models is challenging, because risk assessment is not based on shared risk estimation. This can partially be resolved by focusing on the success factors and concentrating on the external dependencies. In this approach, individual risk vertices are considered as representative of a larger complex IoT system. This advocates a top-down, or goal-oriented modelling approach [31], [46], where success factors are traversing across multiple isolated models. The paradigm can provide a real-time statistical assessment of cyber risk of all the entities in the model. The dependencies between a dynastic metaphor, such as 'parent', 'child', 'orphan' are explained in the transformational roadmap, can be analysed with computational statistics using a Bayesian analysis engine [60], [61]. 


\subsection{Micro Mort}

Since there is no International IoT Asset Classification (IIoTAC) and no established Key IoT Cyber Risk Factors (KIoTCRF), for the calculations of the new model, we would firstly need to determine the IIoTAC and the KIoTCRF [1], [5]. After the establishment of IIoTAC and KIoTCRF, the new model could be applied to calculate more precise 'willingness to pay' to reduce IoT cyber risk.

\section{Conclusion}

This article combines existing literature and performs comparative, empirical and theoretical analysis of common cyber risk assessment approaches and integrates current standards. The findings present a map of the present initiatives, frameworks, methods and models for assessing the impact of cyber risk. Hence, the article advances the efforts of integrating cyber risk standards and governance and offers a better understanding of a holistic impact assessment approach for IoT cyber risk. This enables visualising the interactions among different sets of cyber security assessment criteria and results with a new design criterion specific for cyber risk from the IoT. The visualisation of cyber risk can be used by practitioners and regulators to inform organisations in this space of best practices. The design principles, with the transformational roadmap, can be applied to assess the impact of cyber compromises and to make cyber security recommendations. The findings are relevant to national and international Industry 4.0 networks, specifically for IoT cyber risk planning.

\subsection{Limitations and further research}

Holistic analysis of all risk assessment approaches was considered beyond the scope of this study. Additional research is required to integrate the knowledge from other studies. The epistemological framework in this article presents a generic approach to guide researchers and practitioners.

Author Contributions: conceptualization, Dr Petar Radanliev, Prof. David De Roure and Dr Jason Nurse; methodology, Dr Petar Radanliev, Prof. Peter Burnap, Miss Eirini Anthi, Dr Uchenna Ani, Miss La'Treall Madox, Mr Omar Santos, and Dr Rafael Mantilla Montalvo; validation, Dr Petar Radanliev, Miss La'Treall Madox, Mr Omar Santos and Dr Rafael Mantilla Montalvo.; formal analysis, Dr Petar Radanliev and Dr Uchenna Ani.; investigation, Prof. David De Roure and Dr Petar Radanliev; resources, Dr Petar Radanliev and Dr Rafael Mantilla Montalvo.; data curation, Dr Petar Radanliev and Dr La'Treall Madox.; writing-original draft preparation, Dr Petar Radanliev; writing-review and editing, Dr Petar Radanliev; visualization, Dr Petar Radanliev; supervision, Prof.David De Roure and Dr Rafael Mantilla Montalvo; project administration, Dr Petar Radanliev, Prof. David De Roure and Dr Rafael Mantilla Montalvo; funding acquisition, Dr Petar Radanliev and Prof. David De Roure.

Funding: This research was funded by the UK EPSRC with project [grant number EP/N02334X/1 and EP/N023013/1] and by the Cisco Research Centre [grant number 2017-169701 (3696)].

Acknowledgments: Sincere gratitude to the Fulbright Commission for supporting this project with the Fulbright Visiting Fellowship at MIT.

Conflicts of Interest: The authors declare no conflict of interest.

\section{References:}

[1] P. Radanliev, D. De Roure, R. Nicolescu, M. Huth, R. M. Montalvo, S. Cannady, and P. Burnap, “Future developments in cyber risk assessment for the internet of things," Comput. Ind., vol. 102, pp. 14-22, Nov. 2018.

[2] IBM, “2016 Cyber Security Intelligence Index infographic for Healthcare,” 2016.

[3] Deloitte, "Cyber security: everybody's imperative A guide for the C-suite and boards on guarding against cyber risks," Ontario, 2017. 
[4] J. R. C. Nurse, P. Radanliev, S. Creese, and D. De Roure, “Realities of Risk: 'If you can't understand it, you can't properly assess it!': The reality of assessing security risks in Internet of Things systems," in Living in the Internet of Things: Cybersecurity of the IoT - 2018, 2018, pp. 1-9.

[5] P. Radanliev, D. De Roure, S. Cannady, R. M. Montalvo, R. Nicolescu, and M. Huth, “Economic impact of IoT cyber risk - analysing past and present to predict the future developments in IoT risk analysis and IoT cyber insurance," in Living in the Internet of Things: Cybersecurity of the IoT - 2018, 2018, vol. 2018, no. CP740, p. 3 (9 pp.)-3 (9 pp.).

[6] K. Carruthers, "Internet of Things and Beyond: Cyber-Physical Systems - IEEE Internet of Things," IEEE Internet of Things, Newsletter, 2014, 2016.

[7] D. DiMase, Z. A. Collier, K. Heffner, and I. Linkov, "Systems engineering framework for cyber physical security and resilience," Environ. Syst. Decis., vol. 35, no. 2, pp. 291-300, 2015.

[8] P. Radanliev, “Green-field Architecture for Sustainable Supply Chain Strategy Formulation,” Int. J. Supply Chain Manag., vol. 4, no. 2, Jun. 2015.

[9] P. Radanliev, "Architectures for Green-Field Supply Chain Integration,” J. Supply Chain Oper. Manag., vol. 13, no. 2, Sep. 2015.

[10] P. Radanliev, "Engineering Design Methodology for Green-Field Supply Chain Architectures Taxonomic Scheme," J. Oper. Supply Chain Manag., vol. 8, no. 2, pp. 52-66, Dec. 2015.

[11] P. Radanliev, "Supply Chain Systems Architecture and Engineering Design: Green-field Supply Chain Integration," Oper. Supply Chain Manag. An Int. J., vol. 9, no. 1, 2016.

[12] P. Radanliev, H. Rowlands, and A. Thomas, "Supply Chain Paradox: Green-field Architecture for Sustainable Strategy Formulation," in Cardiff: Sustainable Design and Manufacturing 2014, Part 2, International Conference, 2014, pp. 839-850.

[13] World Economic Forum, "Partnering for Cyber Resilience Towards the Quantification of Cyber Threats," Geneva, 2015.

[14] R. Koch and G. Rodosek, Proceedings of the 15th European Conference on Cyber Warfare and Security : ECCWS 2016 : hosted by Universitat der Bundeswehr, Munich, Germany 7-8 July 2016. 2016.

[15] K. Ruan, "Introducing cybernomics: A unifying economic framework for measuring cyber risk," Comput. Secur., vol. 65, pp. 77-89, 2017.

[16] M. A. Roumani, C. C. Fung, S. Rai, and H. Xie, "Value Analysis of Cyber Security Based on Attack Types," ITMSOC Trans. Innov. Bus. Eng., vol. 01, pp. 34-39, 2016.

[17] R. Anderson and T. Moore, "The Economics of Information Security," Sci. AAAS, vol. 314, no. 5799, pp. 610-613, 2006.

[18] L. A. Gordon and M. P. Loeb, "The economics of information security investment," ACM Trans. Inf. Syst. Secur., vol. 5, no. 4, pp. 438-457, Nov. 2002.

[19] G. Rodewald and Gus, "Aligning information security investments with a firm's risk tolerance," in Proceedings of the 2nd annual conference on Information security curriculum development - InfoSecCD '05, 2005, p. 139.

[20] J. Nurse, S. Creese, and D. De Roure, "Security Risk Assessment in Internet of Things Systems," IT Prof., vol. 19, no. 5, pp. 20-26, 2017.

[21] A. Giordano, G. Spezzano, and A. Vinci, "A Smart Platform for Large-Scale Cyber-Physical Systems," Springer International Publishing, 2016, pp. 115-134.

[22] P. C. Evans and M. Annunziata, "Industrial Internet: Pushing the Boundaries of Minds and Machines," General Electric, 2012.

[23] P. Marwedel and M. Engel, “Cyber-Physical Systems: Opportunities, Challenges and (Some) Solutions," Springer International Publishing, 2016, pp. 1-30.

[24] L. Wang, M. Törngren, and M. Onori, "Current status and advancement of cyber-physical systems in manufacturing," J. Manuf. Syst., vol. 37, pp. 517-527, Oct. 2015.

[25] P. Leitão, A. W. Colombo, and S. Karnouskos, "Industrial automation based on cyber-physical systems technologies: Prototype implementations and challenges," Comput. Ind., vol. 81, pp. 11-25, 2016.

[26] A. Sangiovanni-Vincentelli, W. Damm, and R. Passerone, "Taming Dr. Frankenstein: Contract-Based Design for Cyber-Physical Systems * g," Eur. J. Control, vol. 18, pp. 217-238, 2012.

[27] P. Radanliev, D. C. De Roure, J. R. C. Nurse, P. Burnap, E. Anthi, U. Ani, L. Maddox, O. Santos, and R. M. Montalvo, "Definition of Internet of Things (IoT) Cyber Risk - Discussion on a Transformation Roadmap for Standardisation of Regulations, Risk Maturity, Strategy Design and Impact Assessment," 2018. 
[28] P. Radanliev, D. De Roure, J. R. C. Nurse, R. Nicolescu, M. Huth, S. Cannady, and R. M. Montalvo, “New developments in Cyber Physical Systems, the Internet of Things and the Digital Economy - discussion on future developments in the digital economy," 2018.

[29] P. Radanliev, D. De Roure, J. R. C. Nurse, R. Nicolescu, M. Huth, S. Cannady, and R. M. Montalvo, “Cyber risk impact assessment - discussion on assessing the risk from the IoT to the digital economy," Oxford, 2018.

[30] P. Radanliev, D. C. De Roure, J. R. C. Nurse, P. Burnap, E. Anthi, U. Ani, L. Maddox, O. Santos, and R. M. Montalvo, "Cyber risk from IoT technologies in the supply chain - discussion on supply chains decision support system for the digital economy," 2018.

[31] P. Radanliev and D. C. De Roure, "Decision support system for visualising cyber risk from IoT technologies in supply chains," 2019.

[32] NIST, “Update to Cybersecurity Framework I NIST," National Institute of Standards and Technology, U.S. Department of Commerce, 2017. [Online]. Available: https://www.nist.gov/newsevents/news/2017/12/update-cybersecurity-framework. [Accessed: 31-Mar-2018].

[33] M. Barrett, J. Marron, V. Yan Pillitteri, J. Boyens, G. Witte, and L. Feldman, “Draft NISTIR 8170, The Cybersecurity Framework: Implementation Guidance for Federal Agencies," Maryland, 2017.

[34] FIRST, “CVSS v3.0 Specification Document.” [Online]. Available: https://www.first.org/cvss/specificationdocument\#8-4-Metrics-Levels. [Accessed: 03-Oct-2017].

[35] CVSS, “Common Vulnerability Scoring System SIG," FIRST.org, 2017. [Online]. Available: https://www.first.org/cvss/. [Accessed: 26-Dec-2017].

[36] R. Shaw, V. Takanti, T. Zullo, M. Director, and E. Llc, "Best Practices in Cyber Supply Chain Risk Management Boeing and Exostar Cyber Security Supply Chain Risk Management Interviews," 2017.

[37] CMMI, "What Is Capability Maturity Model Integration (CMMI)®? | CMMI Institute," CMMI Institute, 2017. [Online]. Available: http://cmmiinstitute.com/capability-maturity-model-integration. [Accessed: 26Dec-2017].

[38] FAIR, “Quantitative Information Risk Management I The FAIR Institute," Factor Analysis of Information Risk, 2017. [Online]. Available: http://www.fairinstitute.org/. [Accessed: 26-Dec-2017].

[39] RiskLens, “Risk Analytics Platform I FAIR Platform Management," 2017. [Online]. Available: https://www.risklens.com/platform. [Accessed: 26-Dec-2017].

[40] FAIR, “What is a Cyber Value-at-Risk Model?," 2017. [Online]. Available: http://www.fairinstitute.org/blog/what-is-a-cyber-value-at-risk-model. [Accessed: 26-Dec-2017].

[41] ISO, "ISO - International Organization for Standardization," 2017. [Online]. Available: https://www.iso.org/home.html. [Accessed: 26-Dec-2017].

[42] P. Radanliev, D. C. De Roure, J. R. C. Nurse, R. M. Montalvo, and P. Burnap, "The Industrial Internet-ofThings in the Industry 4.0 supply chains of small and medium sized enterprises," 2019.

[43] P. Radanliev, D. C. De Roure, J. R. C. Nurse, R. M. Montalvo, and P. Burnap, “Cyber risk impact assessment for the Internet of Things (IoT)," Work. Pap., 2019.

[44] P. Radanliev, C. D. De Roure, .R.C. Nurse, R. Nicolescu, M. Huth, C. Cannady, R. M. Montalvo, D. De Roure, J. R. C. Nurse, R. Nicolescu, M. Huth, S. Cannady, and R. M. Montalvo, "Integration of Cyber Security Frameworks, Models and Approaches for Building Design Principles for the Internet-of-things in Industry 4.0," in Living in the Internet of Things: Cybersecurity of the IoT - 2018, 2018, vol. 2018, no. CP740, p. 41 (6 pp.)-41 (6 pp.).

[45] P. Radanliev, D. De Roure, R. Nicolescu, and M. Huth, "The Impact of Cyber Physical Systems and Internet of Everything on Production Economics for the Industry 4.0: A Reference Architecture," 2019.

[46] P. Radanliev, D. C. De Roure, J. R. C. Nurse, P. Burnap, E. Anthi, U. Ani, O. Santos, and R. M. Montalvo, "Definition of Cyber Strategy Transformation Roadmap for Standardisation of IoT Risk Impact Assessment with a Goal-Oriented Approach and the Internet of Things Micro Mart," 2019.

[47] P. Radanliev, D. C. De Roure, J. R. C. Nurse, R. M. Montalvo, P. Burnap, D. C. De Roure, J. R. C. Nurse, R. M. Montalvo, and Stacy Cannady, “Design principles for cyber risk impact assessment from Internet of Things (IoT)," 2018.

[48] L. Taylor, P., Allpress, S., Carr, M., Lupu, E., Norton, J., Smith, H. Blackstock, J., Boyes, H., Hudson-Smith, A., Brass, I., Chizari, D. Cooper, R., Coulton, P., Craggs, B.,Davies, N., De Roure, B. Elsden, M., Huth, M., Lindley, J., Maple, C., Mittelstadt, A. Nicolescu, R., Nurse, J., Procter, R., Radanliev, P., Rashid, R. Sgandurra, D., Skatova, A., Taddeo, M., Tanczer, L., Vieira-Steiner, T. Watson, J.D.M., Wachter, S., 
Wakenshaw, S., Carvalho, G., and P. S. R.J., Westbury, "Internet of Things realising the potential of a trusted smart world," London, 2018.

[49] R. Nicolescu, M. Huth, P. Radanliev, D. De Roure, and R. Nicolescu, “Mapping the values of IoT," J. Inf. Technol., pp. 1-16, Mar. 2018.

[50] L. Axon, B. Alahmadi, J. R. C. Nurse, M. Goldsmith, and S. Creese, "Sonification in Security Operations Centres: What do Security Practitioners Think?," in Proceedings of the Workshop on Usable Security (USEC) at the Network and Distributed System Security (NDSS) Symposium, 2018, pp. 1-12.

[51] J. Eggenschwiler, I. Agrafiotis, and J. R. Nurse, “Insider threat response and recovery strategies in financial services firms," Comput. Fraud Secur., vol. 2016, no. 11, pp. 12-19, Nov. 2016.

[52] P. Radanliev, "A conceptual framework for supply chain systems architecture and integration design based on practice and theory in the North Wales slate mining industry," British Library, 2014.

[53] P. Radanliev, “Green-field Architecture for Sustainable Supply Chain Strategy Formulation,” Int. J. Supply Chain Manag., vol. 4, no. 2, pp. 62-67, 2015.

[54] A. Laugé, J. Hernantes, and J. M. Sarriegi, “Critical infrastructure dependencies: A holistic, dynamic and quantitative approach," Int. J. Crit. Infrastruct. Prot., vol. 8, pp. 16-23, 2015.

[55] M. Bilal, "A Review of Internet of Things Architecture, Technologies and Analysis Smartphone-based Attacks Against 3D printers," arXiv Prepr. arXiv1708.04560, pp. 1-21, 2017.

[56] P. Kotzanikolaou, M. Theoharidou, and D. Gritzalis, "Cascading Effects of Common-Cause Failures in Critical Infrastructures," in Critical Infrastructure Protection VII, Series Vol., vol. 417, no. 2003, J. Butts and S. Shenoi, Eds. Berlin Heidelberg: Springer Berlin Heidelberg, 2013, pp. 171-182.

[57] R. Bloomfield, L. Buzna, P. Popov, K. Salako, and D. Wright, Stochastic modelling of the effects of interdependencies between critical infrastructure, vol. 6027 LNCS. Springer Berlin Heidelberg, 2010.

[58] P. Zhang and S. Peeta, "A generalized modeling framework to analyze interdependencies among infrastructure systems," Transp. Res. Part B Methodol., vol. 45, no. 3, pp. 553-579, 2011.

[59] G. Stergiopoulos, P. Kotzanikolaou, M. Theocharidou, G. Lykou, and D. Gritzalis, "Time-based critical infrastructure dependency analysis for large-scale and cross-sectoral failures," Int. J. Crit. Infrastruct. Prot., vol. 12, pp. 46-60, 2016.

[60] M. D. Weinberg, “Computational statistics using the Bayesian Inference Engine," Mon. Not. R. Astron. Soc., vol. 434, no. 2, pp. 1736-1755.

[61] K. M. Hanson and G. S. Cunningham, THE BAYES INFERENCE ENGINE. 1996. 